\title{
Eficácia psicoterapêutica: terapia de família e o efeito "Dodô"
}

\author{
Orestes Diniz Neto \\ Universidade Federal de Minas Gerais \\ Terezinha Féres-Carneiro \\ Pontifícia Universidade Católica do Rio de Janeiro
}

\begin{abstract}
Resumo
Este trabalho tem por objetivo rever, de modo crítico, os estudos sobre eficácia psicoterapêutica dos modelos e das escolas de terapia em geral e de terapia de família em particular, desenvolvidos sobretudo nos EUA. Discute-se o desenvolvimento do conceito de eficácia psicoterapêutica. Aborda-se o impacto da hipótese “Dodô" (Luborsky, Singer \& Luborsky, 1975), que se refere à similitude de resultados dos métodos psicoterapêuticos. São revistos os resultados de estudos meta-estatísticos e discutidas suas implicações epistemológicas e metodológicas, relacionando-as com o desenvolvimento do campo da psicoterapia. Constata-se a necessidade do desenvolvimento de pesquisas que envolvam métodos mistos, quantitativos e qualitativos.
\end{abstract}

Palavras-chave: psicoterapia; terapia de família; eficácia; epistemologia; metodologia

\begin{abstract}
Psychotherapeutic efficacy: family therapy and the "Dodô" effect. This paper aims at reviewing, in a critical manner, the studies on the psychotherapeutic efficacy of therapeutic models and approaches in general and the family psychotherapy in particular. The development of the psychotherapy efficacy and efficiency concept is discussed. The impact of Dodo hypotheses (Luborsky, Singer \& Luborsky, 1975), which refers to the similar results of the psychotherapeutic methods, is considered. The results of the meta-statistical studies are reviewed, and their epistemological and methodological implications are discussed, relating them to the development of the psychotherapy. The need of a research development that comprises qualitative and quantitative methodologies is verified.
\end{abstract}

Key words: psychotherapy; family therapy; efficacy; efficiency; epistemology; methodology

$\mathrm{E}$ ste trabalho pretende rever os principais estudos sobre eficácia psicoterapêutica, tal como compreendida pela American Psychological Association (APA), das escolas e dos modelos de terapia de família e de casal, discutindo suas implicações metodológicas e epistemológicas para o desenvolvimento deste campo. A discussão destes aspectos é relevante, não só pela necessidade de balizamento dos estudos sobre eficácia e eficiência psicoterapêutica, mas também pela pressão da demanda social, em especial as comparações com outros métodos de tratamento, tais como os psicofarmacológicos e outros surgidos com a chamada Psiquiatria Biológica, feitas pelas companhias de seguro-saúde nos EUA e pelos laboratórios farmacêuticos. Cabe ressaltar que estes estudos foram desenvolvidos inicialmente e com grande predominância nos EUA e expandiram-se posteriormente para outros países da Europa (Chambless \& Ollendick, 2001; Kopta, Luguer, Sanders, \& Howard, 1999). Não foram encontrados estudos comparativos sobre eficácia psicoterapêutica desenvolvidos no Brasil.

Uma das respostas a esta pressão, com fortes repercussões metodológicas e epistemológicas, foi a criação, pela American Psychological Association (APA), por meio da Divisão de Psicologia Clínica (Divisão 12), em 1993, de uma força tarefa com vistas a discutir critérios para a validação de modelos terapêuticos "empiricamente fundamentados", e a elaboração de uma lista de métodos e modelos terapêuticos aprovados por estes critérios (Chambless \& Ollendick, 2001).

Um website, com o título A guide to beneficial psychotherapy, foi criado pela Divisão 12 da APA, com os objetivos de disseminar os critérios para avaliação de modelos psicoterapêuticos empiricamente validados, divulgar uma lista daqueles aprovados por esses critérios, e orientar o público na escolha de métodos de tratamento psicoterapêutico. 
Para responder às necessidades de informação do público sobre os benefícios da psicoterapia, este website foi desenvolvido pelo Comitê sobre Ciência e Prática da Sociedade de Psicologia Clínica, uma divisão da APA, para prover uma breve descrição de várias psicoterapias que têm encontrado padrões científicos básicos de efetividade (Society of Clinical Psychology, 2003). ${ }^{1}$

As sucessivas avaliações feitas por essa força tarefa abordaram diferentes focos de tratamento, como o de adultos, de crianças, de família e de casal, publicando uma lista de métodos de tratamentos psicoterapêuticos aprovados (Chambless \& Olendick, 2001).

Surgiram, assim, diversas questões, pois, ao referenciar a escolha de modelos de tratamento que seriam efetivos e, portanto, aceitáveis pelas companhias de seguro e o público em geral, a APA padronizou um método de validação, ou seja, o estudo comparativo controlado (Chambless \& Ollendick, 2001; Kopta et al., 1999):

Nós demarcamos critérios estritamente científicos para a avaliação das psicoterapias presentes aqui, apoiados nos dados de estudos cuidadosamente controlados. Não nos apoiamos sobre opiniões de pacientes, opiniões de profissionais, informação obtida de estudos de pesquisa não controlados, ou outras fontes de informação não controladas. Dados coletados por cientistas em estudos controlados fornecem a mais objetiva informação disponível sobre os benefícios da psicoterapia (Society, 2003).

Entretanto, é notável nesta lista a ausência de sistemas e abordagens normalmente consagrados na prática clínica, ao que a APA responde:

Algumas psicoterapias bem conhecidas não aparecem aqui. Usualmente, porque elas não foram submetidas aos tipos de estudos controlados descritos acima, mais do que por essas psicoterapias terem sido consideradas ineficazes ou danosas. (Society, 2003)

Com o objetivo de rever os estudos sobre eficácia em psicoterapia, consultou-se a base de dados PsycINFO, em maio de 2003, com os critérios de busca (palavras-chave): efficacy, therapy, e family. Foram selecionados os estudos de revisão sobre eficácia em psicoterapia de modo geral e de família em particular, do período de 1960 a 2003.

\section{Estudos sobre eficácia da psicoterapia}

Desde o início do século XX, praticamente a partir do aparecimento de escolas e métodos de tratamento psicoterapêutico, iniciaram-se discussões sobre indicações e eficácia de métodos de tratamento (Garsk \& Lynn, 1985; Marx \& Hillix, 1963/1998). O método de avaliação do tratamento psicoterapêutico era unicamente o método clínico de estudo de caso, que muitos clínicos consideram, ainda hoje, como o único válido para se avaliar um dado procedimento.

Eysenck, em 1952, deu início às discussões sobre a validade dos métodos terapêuticos, propondo o uso de um método comparativo experimental para um teste de eficácia, uma vez que se considerava que pacientes melhorariam de seu mal-estar psicológico mais rapidamente quando submetidos a um tratamento psicoterapêutico. Comparou clientes tratados em terapia com clientes similares que não foram tratados, limitando-se a clientes "neuróticos". Utilizou dados sobre resultados de tratamento em psicoterapia de 24 estudos não controlados publicados até então. Como grupo comparativo, Eysenck (1952) utilizou dois estudos não controlados, um de Ladis (1937), sobre pacientes neuróticos tratados em hospitais estaduais de Nova York e que receberam unicamente os cuidados normais como pacientes internos, e o outro de Denker (1946), sobre pacientes neuróticos que haviam feito demanda por incapacidade mental em companhias de seguro e que foram tratados, por médicos de prática geral, como pacientes externos. As conclusões de Eysenck (1952) foram que:

Os pacientes tratados por meio de psicanálise melhoraram até um índice de $44 \%$, os pacientes tratados ecleticamente melhoraram em $64 \%$; os pacientes tratados sob custódia ou por médicos gerais melhoraram até um índice de $72 \%$. Parece existir, portanto, uma correlação inversa entre recuperação e psicoterapia. (p. 322)

Eysenck recebeu uma avalanche de críticas e réplicas (Bergin, 1963; Kiesler, 1966; Luborsky, 1954; Malan, 1973; Rosensweig, 1954) que giraram sobre o método e os cálculos errôneos que ele havia realizado. Porém, nenhum crítico chegou a afirmar que os trabalhos revisados por Eysenck (1952) haviam demonstrado a eficácia da psicoterapia. Este estudo pioneiro, em retrospectiva, levou ao desenvolvimento e aperfeiçoamento de metodologias com o objetivo de estudar a eficácia da psicoterapia.

Durante a década de 70, surgiu como padrão na investigação de eficácia em psicoterapia, o estudo comparativo controlado que se desenvolveu em desenhos experimentais sofisticados. Os critérios que passaram a caracterizar uma investigação bem projetada em psicoterapia eram, a partir de então, para Garsk e Lynn (1985):

- grupos homogêneos de clientes com problemas específicos e idênticos, distribuídos aleatoriamente sob condições experimentais;

- terapeutas igualados quanto a variáveis críticas como experiência, prática formal de certo modelo;

- grupos de controle cuidadosamente desenhados para rejeitar hipóteses alternativas tais como efeitos da atenção, expectativa e contato pessoal;

- múltiplas medidas de mudança, incluindo os aspectos comportamental, cognitivo e afetivo;

- medidas realizadas antes e depois de um período de tratamento e com acompanhamento, isto é, certo período de tempo após.

A pergunta sobre eficácia havia, então, evoluído, convertendo-se em: que tratamento, nas mãos de quem, para quem, com que problema, sob que circunstâncias, será mais efetivo?

Duas décadas depois dos primeiros trabalhos de Eysenck (1952), os dados provenientes de investigação haviam demonstrado que certas psicoterapias, realizadas por certos terapeutas, com certos pacientes, eram demonstravelmente mais eficazes em comparação com grupos de controle sem 
tratamento (Garsk \& Lynn, 1985; Stricker, 1992). Eficácia passara a significar a diferença de resultado obtida entre clientes, com quadros "semelhantes”, tratados e não-tratados. Isso levou ao desenvolvimento de desenhos experimentais sofisticados, possibilitando a realização de diversos estudos com resultados significativos. Numerosos métodos de tratamento passaram a demonstrar sua eficácia, trazendo à tona uma nova pergunta: qual abordagem é a mais eficaz? (Garsky \& Lynn, 1985). No centro desta questão parecia haver uma busca por uma definição paradigmática, pois, pela primeira vez, parecia possível verificar empiricamente as conseqüências das teorias através das técnicas psicoterapêuticas delas decorrentes.

Entretanto, como comparar os resultados obtidos por diversos estudos? Alguns, como o estudo da Temple University Health Sciences Center (Sloane, Staples, Cristol, Yorkston \& Whipple, 1975), que comparou diretamente dois métodos de psicoterapia - a abordagem comportamental e a psicanalítica -, revelaram que ambas as abordagens eram igualmente efetivas quando comparadas a um grupo de controle sem tratamento. Mas, em sua maioria, os estudos eram realizados comparando um método de tratamento com um grupo de controle de pacientes em uma lista de espera para tratamento, e utilizando critérios e métodos de avaliação da mudança terapêutica em consonância com as teorias da abordagem psicoterapêutica, tornando difícil qualquer possibilidade de comparação entre estudos.

Os trabalhos de revisão debatiam sobre um número crescente de demonstrações de eficácia. E estas análises, embora parecessem oferecer um critério para demonstrar a eficácia de uma determinada abordagem e selecionar um paradigma, eram de início enviesadas pela identificação do autor com uma abordagem e, na verdade, muito pouco auxiliaram na questão. Os padrões típicos de análise e comparação eram:

Análise redutiva: na qual os autores realizavam interpretações dos resultados e técnicas segundo os princípios explicativos de sua própria escola (Lazarus, 1980; Wachtel, 1981).

Análise paralelista: pretendia descobrir paralelos entre as diversas abordagens em psicoterapia, tanto ao nível conceitual como técnico, em uma abordagem mais diplomática, que produtiva. Foram mais comuns comparações entre psicanálise e terapia comportamental (Marks \& Gelder, 1966; Marmor, 1971).

Abordagem eclética: neste grupo o foco principal era nas técnicas consideradas como eficazes e que deveriam ser escolhidas conforme a situação e o cliente (Allport, 1960; Burton, 1974/1978).

Modelo em matriz: outra tentativa de síntese que se orientava pela hipótese de que as diferentes escolas e métodos se referiam a diferentes níveis de observação da realidade, sendo, desta forma, complementares. Neste caso, o modelo proposto tomava a forma de uma matriz, sendo considerado como critério para utilização de cada um dos modelos principais em psicoterapia o foco de uma dada situação (Grof, 1985/ 1988; Weil, 1978).

Luborsky, Singer e Luborsky, em 1975, realizaram uma tentativa de superar os métodos qualitativos de análise, pro- curando quantificar a comparação entre a efetividade dos tratamentos das diversas escolas. Utilizaram um método comparativo que envolvia o uso de uma tabela de classificação dos resultados obtidos por um método de tratamento, em diversos estudos, em situações reais de tratamento. Seu procedimento incluiu a avaliação de cada estudo segundo critérios de qualidade. Os estudos medíocres foram eliminados. Realizaram, a seguir, inúmeras comparações: terapia breve versus terapia de longa duração; terapia de grupos versus terapia individual; terapia comportamental versus psicanálise; terapia versus ausência de terapia (grupo de controle). A seguir, atribuiu-se a seguinte pontuação: $(+)$ se o tratamento era significativamente mais efetivo que o outro com o qual era comparado; (-) se o tratamento era menos efetivo que o par de comparação; e (0) se ambos os tratamentos eram iguais, isto é, se não havia diferença estatística significativa.

Foram então somadas as pontuações entre os vários estudos, obtendo-se uma matriz de pontuações. Por exemplo, se uma técnica terapêutica era comparada com um grupo de controle em dez estudos diferentes, e oito pontuações eram positivas, uma negativa e uma neutra, a pontuação na matriz seria (8$0-1)$ e sugeria que a terapia havia sido comparada com os grupos controle e era considerada efetiva. Através desta técnica, evidenciou-se que várias formas de terapia eram efetivas, mas não muito diferentes entre si. Este resultado ficou conhecido como o veredicto "Dodô”, em uma referência ao livro de Lewis Caroll, Alice no país das maravilhas, no qual o pássaro Dodô, após uma corrida, proclama: “Every body has won, and all must have prizes” (Luborsky et al., 1975, p. 1006).

Este intrigante resultado foi inicialmente atribuído ao método de análise, pois o procedimento apresentava algumas dificuldades metodológicas, uma vez que não eliminava a seletividade por parte dos avaliadores, que poderiam escolher um estudo em detrimento de outro, por motivos subjetivos. Além disso, a análise na realidade não foi estatística, por se restringir aos dados da matriz de pontuação, não levando em conta a magnitude da diferença dos resultados nas comparações.

Smith, Glass e Miller (1980) sugeriram, como alternativa, um procedimento quantitativo para integrar os resultados de estudos sobre eficácia em psicoterapia. Utilizaram dados que são publicados normalmente em artigos tais como média, razão $t$, razão $F$ ou outro parâmetro estatístico. O procedimento incluía uma meta-análise estatística, na qual o parâmetro estatístico principal foi a magnitude de efeito, que era obtida ao se dividir a diferença média de um grupo tratado e um grupo de controle pelo desvio padrão do grupo de controle. Deste modo, se obtém a magnitude de efeito, que é uma média padronizada da diferença e pode ser utilizada nas comparações de um grande número de estudos, que utilizam procedimentos e medidas diferentes. Tal conceituação permitia a comparação de medidas tomadas por diferentes métodos de avaliação, respeitando os critérios de mensuração de cada abordagem. Convém ressaltar que o método de comparação meta-estatístico, no caso específico de avaliação de magnitude de efeito, é uma comparação de resultados obtidos e não de processos.

Foram analisados 475 estudos sobre resultados em psicoterapia e realizados mais de 1766 cálculos de magnitude 
de efeito. Os resultados lançaram luzes em diversas questões sobre eficácia, sendo as seguintes as principais conclusões: (1) As diversas formas de terapia tiveram um resultado positivo. A média da magnitude de efeito foi 0,85 , chegando a 0,93 quando se eliminaram os tratamentos placebo e técnicas de aconselhamento indiferenciadas.

(2) Não houve grau diferenciado de melhora quando se compararam terapias de distintas orientações teóricas, como psicanálise, comportamental, cognitivista, centrada no cliente. Nem as diversas modalidades - verbal, comportamental, ou expressiva - obtiveram resultados diferenciados. As comparações simples e não controladas sugeriram que a hipnoterapia, a dessensibilização sistemática e a terapia cognitivista eram mais efetivas. Porém, esta diferença desaparece se levarmos em conta o tipo de cliente e de medições de resultados. As várias formas de terapia, independente de orientação, foram mais efetivas com clientes depressivos, com fobias simples, ou com casos análogos.

(3) As intervenções breves versus de longo prazo, de grupo versus individual, e de terapeutas experientes versus novatos obtiveram resultados estatisticamente similares.

(4) Os resultados positivos da psicoterapia diminuem dois anos após o tratamento; a média da magnitude de efeito cai para 0,50. Além disto, cerca de $9 \%$ dos resultados terapêuticos são negativos, sendo este resultado similar para todas as formas de terapia estudadas.

Dados os resultados sobre as pesquisas de eficácia no campo das psicoterapias, Garske e Lynn (1985) chegaram a uma conclusão em dois níveis.

Primeiro, muitos tipos e formas de psicoterapia são modestamente efetivas. Segundo, em termos do grau e de extensão da eficácia, as psicoterapias parecem ser mais parecidas que diferentes. A sofisticação emergente neste campo de investigação poderia muito bem proporcionar dados que alterem estas conclusões no futuro. Por hora, apesar das pretensões de diversos partidários e críticos, a avaliação que acabamos de apresentar é parcimoniosa e justa. (p. 631) ${ }^{2}$

Dez anos após, em uma revisão sobre o tema, Miller, Hubble e Duncan (1995), ao avaliar o campo de pesquisa sobre eficácia das psicoterapias, notam que apesar de inúmeros desenvolvimentos em técnicas de pesquisa, o quadro geral não se alterou, notando que até mesmo formas emergentes de psicoterapia, como terapia cognitiva e terapia de família e de casal, também demonstraram sua eficácia. Tal panorama não se alterou no início do século XXI (Chambless \& Ollendick, 2001).

\section{A eficácia da terapia de família}

Com o desenvolvimento e divulgação de métodos de tratamento centrados na família e no casal, durante as décadas de 1960 e 1970, alguns estudos sobre sua eficácia foram realizados. Porém, apenas na década de 1980 seriam realizados estudos meta-estatísticos, uma vez que modelos e abordagens de terapia de família haviam sido avaliados, usando o método de estudo comparativo controlado. Estudos meta- estatísticos, como os de Hahlweg e Markman (1988), e de Hazelrigg, Cooper e Borduin (1987), demonstraram a eficácia geral dessas formas de tratamento sem, contudo, pesquisar outros aspectos.

Shadish et al. (1993) aperfeiçoaram o método de comparação, procurando examinar diferenças entre métodos de diversas orientações teóricas, bem como diferenças de resultados entre terapia individual e de casal. Utilizaram 163 estudos que haviam sido publicados, entre 1963 e 1988, sobre teste de eficácia em psicoterapia, e incluíram em sua análise teses e dissertações sobre o tema, que não haviam sido consideradas nos estudos anteriores.

A análise destes estudos seguiu os padrões recomendados pelo National Research Council (1992), não utilizando estudos quase-experimentais, mas apenas os randomizados. Suas conclusões foram:

- clientes tratados em terapia de casal e terapia de família têm melhoras superiores aos indivíduos não tratados nos póstestes, sendo a estimativa de magnitude de efeito similar aos das meta-análises anteriores;

- certas abordagens de tratamento parecem ter resultados superiores em algumas comparações realizadas em estudos do tipo grupo experimental e de controle não ajustados, mas quando são realizadas correções na análise da regressão estas diferenças desaparecem;

- diferenças similares aparecem em estudos de comparação entre diferentes orientações teóricas de tratamento, mas também desaparecem, ao ser realizada a análise de regressão;

- se todos os tratamentos são igualmente bem projetados, implementados, medidos e relatados, não se encontram diferenças significativas entre as abordagens;

- houve uma consistente falha das terapias humanísticas em alcançar resultados positivos em qualquer análise.

Pinsof e Wynne (1995a, 1995b) revisaram grande parte dos estudos sobre eficácia de terapia de família e de casal publicados até então, encontrando seis características nos estudos bem controlados sobre eficácia terapêutica:

- ocorriam em ambiente clínico controlado, como laboratórios de pesquisa;

- focavam um problema ou desordem psiquiátrica específico e definível;

- envolviam pelo menos dois grupos ou condições: um experimental, que recebia o tratamento, e um grupo de controle que em uma lista de espera recebia um tratamento alternativo; - os grupos eram randômicos;

- os tratamentos eram especificados e dirigidos por manuais, sendo a performance do terapeuta monitorada durante o tratamento; - todos os clientes eram avaliados em medidas antes e depois através de avaliações padronizadas. Em experimentos mais recentes uma avaliação follow-up era realizada, constituindo uma terceira medida.

Pinsof e Wynne (1995a, 1995b) consideraram como critério de validação a existência de pelo menos dois estudos independentes com resultados significativos, concomitantemente com a ausência de resultados negativos em qualquer outro estudo, tendo chegado às seguintes conclusões: 
(1) Terapia de família tem resultados melhores do que abordagem individual para: esquizofrenia, alcoolismo em adultos, adição em drogas em adultos e adolescentes, desordem de conduta em adolescentes, anorexia em adolescentes, autismo infantil, agressões e dificuldades em atenção, no transtorno de déficit de atenção/hipermotividade, processos demenciais, fatores de risco cardiovascular. Terapia de família é ainda melhor do que a ausência de tratamento nos casos anteriores e, ainda, para: obesidade na adolescência, anorexia na adolescência, desordens de conduta na infância, obesidade infantil, doenças crônicas na infância.

(2) A abordagem de terapia de casal mostrou-se mais efetiva do que a abordagem individual para depressão em mulheres em casamento disfuncional e para casamentos disfuncionais. Evidenciou-se ainda ser melhor do que a ausência de tratamento para todos os casos indicados acima e mais, para obesidade de adultos e hipertensão de adultos (Pinsof \& Wynne, 1995b, 2000).

(3) Não se encontrou nenhum estudo que demonstrasse efeitos adversos da terapia de família e de casal.

(4) Tampouco se evidenciaram dados suficientes para apoiar a superioridade de uma abordagem de terapia de família sobre outras.

(5) Os dados indicaram que a abordagem de casal e família possui um custo efetivo melhor do que o tratamento padrão em hospitais.

(6) Terapia de casal e de família não são suficientes para tratar sozinhas desordens mentais crônicas como, esquizofrenia, desordens afetivas mono e bipolares, adicções, autismo e desordens severas de conduta.

(7) Em todos os casos, em que terapias de família e de casal foram utilizadas em combinação com outros tratamentos, o resultado final foi potencializado.

Tais resultados confirmaram a eficácia geral do tratamento de terapia de casal e família. Porém, isto não significa que não haja limites e restrições a estes estudos, relativos à sua fundamentação epistemológica e metodológica.

\section{Implicações epistemológicas e metodológicas}

Embora os estudos sobre eficácia terapêutica tenham contribuído para demonstrar a utilidade da psicoterapia como modo de tratamento para inúmeros problemas psicológicos de modo inequívoco, segundo o paradigma científico clássico, ainda assim, levantaram diversas críticas. Estas foram fundamentalmente dirigidas não à necessidade de se demonstrar a eficácia das diversas psicoterapias, ou mesmo discriminálas em relação a indicações terapêuticas especificas, mas à metodologia utilizada no estudo da eficácia.

Kiesler, em 1966, já apontava o problema do que chamou mito da uniformidade, que estaria presente em todos os experimentos do tipo pré/pós, considerando que esta metodologia supõe incorretamente que a psicoterapia seria aplicada uniformemente em todos os casos, como se os terapeutas fossem invariáveis e como se os casos também pudessem ser separados em padrões homogêneos. Esta falácia contaminaria, assim, os estudos meta-analíticos, qualquer que fosse sua metodologia, pois são, necessariamente, reali- zados sobre estudos do tipo pré/pós, como nos modelos de estudos comparativos controlados.

Pesquisadores responderam a estas críticas, buscando desenvolver desenhos experimentais, cada vez mais uniformes, com controle tanto dos procedimentos terapêuticos, que passaram a ser orientados por manuais e supervisionados, como do uso do modelo em teste, que passou a ser feito através de avaliações altamente estruturadas dos casos em atendimento, através de gravações e análise das sessões. Tal abordagem, ironicamente, aprofundou ainda mais a distância entre os estudos sobre eficácia e a prática clínica, pois os modelos em teste passaram a ser altamente diferenciados, pelo menos aparentemente. Já na prática clínica, as pesquisas conduzidas nos últimos dez anos têm, invariavelmente, apontado para uma tendência, cada vez mais integrativa e eclética, entre os psicoterapeutas (Pinsof \& Wynne, 1995b, 2000). De fato, cada vez mais, têm sido desenvolvidos modelos multimodais e multidisciplinares, com resultados superiores aos dos tratamentos monomodais (Pinsof \& Wynne, 2000).

Por outro lado, os testes têm sido realizados em ambientes altamente controlados e muitos críticos, usualmente, questionam se tais estudos teriam utilidade na realidade da prática clínica, uma situação bastante diversa de uma clínica de pesquisa. A questão seria, então: esses modelos que demonstraram eficácia são, além disso, eficientes em uma situação real? O conceito de eficiência tem sido usado de diferentes maneiras por diferentes autores. A força tarefa designada pela Divisão 12 (Psicologia Clínica) da APA, estabeleceu que no processo de considerar um modelo terapêutico como empiricamente validado, pesquisas de eficiência deveriam se seguir à fase do estabelecimento da eficácia terapêutica (Chambless \& Ollendick, 2001; Kopta et al., 1999). De acordo com esta definição o teste de eficiência é aquele realizado em uma situação real, com tratamentos de eficácia já demonstrada em um ambiente laboratorial clínico, com o objetivo de testar seu uso na prática clínica cotidiana.

Neste caso, ainda prevalece o critério de uniformidade, que supõe que um tratamento mostrou-se eficaz no teste controlado, e agora cabe adaptar sua suposta uniformidade ao mundo real. Além disto, Gottman e Rushe (1993) chamaram a atenção para alguns mitos presentes em análise de estudos longitudinais quantitativos, no que se refere à mudança, ressaltando a suposição de que dois pontos de medida são adequados para estudar a mudança. Entre dois pontos podem passar diversas curvas e não apenas uma linha reta. Assim, duas medidas arbitrárias no tempo refletiriam apenas as variações nestes momentos, mas não o processo em andamento. Modelos de mudança caóticos podem facilmente mascarar a complexidade de suas funções se realizarmos poucas medidas discretas. Cabe ainda notar que modelos de mudança psicológica podem ser descritos como processos em cascata e não lineares, nas quais uma longa medida de estabilidade antecede o processo de saltos (Hoffman, 1993/1995; Prochaska, Diclements \& Norcross, 1992). Isto implica a necessidade, mesmo na utilização de métodos quantitativos, do uso de projetos de pesquisa mais sofisticados com medidas múltiplas (Gottman \& Rushe, 1993). 
Outra questão importante se refere à compreensão dos fatores envolvidos na mudança terapêutica. A homogeneidade dos resultados alcançados e a impossibilidade de demonstração clara quanto à eficácia de fatores específicos na mudança terapêutica, levaram à elaboração da chamada teoria dos fatores comuns que emprestariam de fato sua eficácia às diversas formas de terapia (Garsk \& Lynn, 1985). Altshuler, em uma revisão realizada em 1989, ressaltou os seguintes fatores inespecíficos: boa relação terapeuta-cliente, aceitação e apoio ao paciente, oportunidade de expressar emoções, rituais terapêuticos que emprestam significado ao problema, uma explanação compreensiva do problema. Embora a teoria dos fatores comuns favorecesse uma interação mais amistosa entre as diversas abordagens, por outro lado promoveu certa paralisação, pois levou a se desconsiderar outros fatores presentes, como as técnicas específicas. Assim, surgiu como conseqüência a sensação de que qualquer esforço de aprimorar modelos e técnicas seria inútil, tendo um efeito mínimo sobre os resultados (Cordioli, 2002). Portanto, é também como uma tentativa de escapar desta situação paralisadora que se deve compreender o esforço de desenvolver novos modelos terapêuticos, explorando as conseqüências de novas teorias. Apenas com a utilização combinada de estudos sobre a eficácia terapêutica e modelos de mudança terapêutica é que serão possíveis avanços significativos no campo das psicoterapias. O uso de metodologias qualitativas parece ser fundamental nesse aspecto. É claro que métodos quantitativos têm utilidade, mas não podem ser considerados como única fonte de dados úteis sobre o processo de mudança. Desde o inicio dos anos 1990, um grande número de autores tem advogado e utilizado métodos quantitativos e mistos para abordar questões ligadas ao processo psicoterapêutico, em especial no campo da família. E uma mudança na direção das metodologias de pesquisa utilizadas parece ter ocorrido, com um aumento substancial do uso de métodos qualitativos e mistos (Helmek \& Sprenkle, 2000; Johnson, 2000). Falkner, Klock e Gale (2002), ao analisarem as tendências de publicações de métodos qualitativos, notam que a publicação de artigos nos periódicos American Journal of Family, Contemporary Family Therapy, Family Process, e Journal of Marital and Family Therapy aumentou significativamente, de 39 artigos publicados na década de 1980 a 1989 para 90 artigos publicados na década de 1990 a 1999. As áreas de maior foco têm sido o processo terapêutico, o divórcio e o relacionamento familiar. Embora este aumento seja significativo, ainda predominam trabalhos de metodologia quantitativa. Hawley, Bailey e Pennick (2000), ao analisarem as pesquisas empíricas publicadas em revistas científicas de terapia de família, de 1994 a 1998, encontraram uma predominância do uso de métodos quantitativos em cerca de 80\% dos 199 artigos analisados.

\section{Reflexões Finais}

Diversas questões emergem dos estudos sobre eficácia terapêutica. É seguro afirmar que psicoterapia de modo geral, como método de tratamento para problemas de ordem psico- lógica, é uma alternativa de eficácia comprovada, em certos modelos terapêuticos. A direção das pesquisas sobre as práticas psicoterapêuticas, em termos de eficácia, tem ocorrido ao redor de modelos terapêuticos específicos para clientes com quadros similares, em situação similar. Assim, é possível validar modelos, mas não escolas, teorias ou métodos terapêuticos em bloco. É notável a ausência de diferenças significativas de resultados, na maior parte dos estudos metaestatísticos, entre as diversas escolas de psicoterapia, podendo-se considerar que não é possível concluir sobre a adequação, maior ou menor, de uma dada escola psicoterápica. Portanto, todas as afirmativas sobre superioridade geral de uma dada abordagem sobre as demais continuam como nãocomprovadas. Todavia, existem irregularidades, que podem até mesmo se revelar profundamente significativas, como a ausência de estudos que comprovem a eficácia das abordagens humanistas no atendimento clínico à família e ao casal (Shadish et al., 1993). Tais irregularidades, se confirmadas, podem indicar que as técnicas humanísticas, centradas no conceito de auto-organização, eficazes no tratamento individual e de grupo, não são suficientes para lidar com os complexos processos de um sistema familiar ou conjugal.

Cabe ressaltar ainda que embora as pesquisas comparativas possam revelar dados sobre a eficácia dos métodos psicoterapêuticos pouco revelam sobre a maneira como são alcançados. Além disto, os modelos são testados em pacotes fechados, como um conjunto de teorias e técnicas que orientam as intervenções dos terapeutas e que, na prática clínica, tendem a variar conforme cada relação terapêutica. Cada caso é único e as relações entre os fatores envolvidos no processo também. Cabe questionar, ainda, se métodos exclusivamente quantitativos podem descrever processos de mudança psicológica. Grande parte do processo de mudança psicológica é de difícil quantificação e pode mesmo não ser quantificável, ainda que seja qualificável. A tendência emergente de utilização de métodos qualitativos vem responder a esta necessidade.

Existe uma grande premência de pesquisas, cuidadosamente planejadas, com claras descrições de seus métodos e análises. As respostas às questões principais do campo da psicoterapia poderão emergir do uso articulado de múltiplas metodologias para abordar os diferentes aspectos envolvidos. Assim, consideramos que estamos agora preparados para caminharmos para o próximo nível, para além do debate sobre a procedência de métodos quantitativos ou qualitativos.

\section{Referências}

Allport, G. W. (1960). The open system in personality theory. Journal of Abnormal Psychology, 61, 301-310.

Altshuler, K. Z. (1989). Will the psychotherapies yield differential results? A look at assumptions in therapy trials. American Journal of Psychotherapy, 43(3), 310-320

Bergin, A. E. (1963). The effects of psychotherapy: negative results revisited. Journal of Counselling Psychology, 10, 244-250.

Burton, B. (1978). Teorias operacionais da personalidade. Rio de Janeiro: Imago. (original em inglês, 1974) 
Chambless, D. L., \& Ollendick, T. H. (2001). Empirically supported psychological interventions; controversies and evidence. Annual Review of Psychology, 52, 685-716.

Cordioli, A. V. (2002). Psicoterapias atuais. Porto Alegre: Artes Médicas.

Decharms, R. J. (1968). Personal causation: the internal affective determinants of behavior. Nova York: Academic.

Denker, P. G. (1946). Results of a treatment of psychoneuroses by the general practitioner. New York State Journal of Medicine, 46, 2146-2166.

Eysenck, H. J. (1952). The effects of psychotherapy: an evaluation. Journal of Consulting Psychology, 16, 319-324.

Falkner, R. A., Klock, K., \& Gale, J. E. (2002). Qualitative research in family therapy: publications trends from 1980 to 1999. Journal of Marital and Family Therapy, 28(1), 69-74.

Garske, J. P., \& Lynn, S. (1985). Hacia un esquema general de la psicoterapia: eficácia, factores comunes e integración. In J. P. Garske, \& S. Lynn (Orgs.), Psicoterapias contemporaneas. Modelos e mitos (pp. 623-647). Bilbao: DDB.

Gottman, J. M., \& Rushe, R. H. (1993). The analysis of change; issues fallacies and new ideas. Journal of Consulting and Clínical Psychology, 61(6), 907-910.

Grof, S. (1988). Além do cérebro. São Paulo: MacGraw-Hill. (original em inglês, 1985)

Hahlweg, K., \& Markman, H. J. (1988). Effectiveness of behavioral marital therapy: empirical status of behavioral techniques in preventing and alleviating marital distress. Journal of Consulting and Clinical Psychology, 56, 440-447.

Hawley, D. R., Bailey, C. E., \& Pennick, K. A. (2000). A content analysis of research in family therapy journals. Journal of Marital and Family Therapy, January, 26(1), 9-26.

Hazelrigg, M. D., Cooper, H. M., \& Borduin, C. M. (1987). Evaluating the effectiveness of family therapies: an integrative review and analysis. Psychological Bulletin, 101, 428-442.

Helmeke, K. B., \& Spreenkle, D. H. (2000). Client's perceptions of pivotal moments in couples therapy: a qualitative study of change in therapy. Journal of Marital and Family Therapy, 26(4), 469-483.

Hoffman, L. (1995). O ciclo de vida familiar e a mudança descontínua. In B. Carter, \& M. Mcgoldrick (Orgs.), As mudanças no ciclo de vida familiar (pp. 84-96). Porto Alegre: Artes Médicas. (original em inglês, 1993)

Johnson, S. (2000). The "coming of age" of couple therapy: a decade review. Journal of Marital and Family Therapy, 26(1), 23-38.

Kiesler, D. J. (1966). Some myths of psychotherapy research and the search for a paradigm. Psychological Bulletin, 65, 110-136.

Kopta, S. M., Luguer R. J., Sanders, S. M., \& Howard, K. I. (1999). Individual psychotherapy outcome and process research: challenges leading to greater turmoil or a positive transition? Annual Review of Psychology, 50, 441-469.

Ladis, C. A. (1937). A statistical evaluation of psychotherapeutic methods. In L. E. Hinsie (Org.), Concepts and problems of psychotherapy (pp. 143-162). Nova York: Columbia University Press.

Lazarus, A. (1980). Toward delineating some causes of change in psychotherapy. Professional Psychology, 11, 863-870.
Luborsky, L. (1954). A note on Eysenck's article "The effects of psychotherapy: an evaluation”. British Journal of Psychology, 45, 129-131.

Luborsky, L., Singer B., \& Luborsky. L. (1975). Comparative studies in psychotherapy: a review of quantitative research. Archives of General Psychiatry, 32, 995-1008.

Malan, D. H. (1973). The outcome problem in psychotherapy research: a historical review. Archives of General Psychiatry, 29, 719-729.

Marks, I., \& Gelder, M. G. (1966). Common ground between behavior therapy and psychodynamic methods. British Journal of Medical Psychology, 39, 11-23.

Marmor, J. (1971). Dynamic psychotherapy and behavior therapy: are they irreconcilable? Archives of General Psychiatry, 29, 22-28.

Marx, M. H., \& Hillix, W. A. (1998). Sistemas e teorias em psicologia. São Paulo: Cultrix. (original em inglês, 1963)

Miller, S., Hubble, M., \& Duncan, B. (1995). No more whistles and bells. Net Worker (Nova York), Março/Abril, 53-63.

National Research Council (1992). Combining information; statistical issues and opportunities for research. Washington, DC: National Academy Press.

Pinsof, W. M., \& Wynne, L. C. (1995a). (Orgs.) Special issue: the effectiveness of marital and family therapy. Journal of Marital and Family therapy, 21(4), 1-7.

Pinsof, W. M., \& Wynne, L. C. (1995b). The efficacy of marital end family therapy: a overview, conclusions and recommendations. Journal of Marital and Family Therapy, 21(4), 585-613.

Pinsof, W. M., \& Wynne, L. C. (2000). Toward progress research: closing the gap between family practice and research. Journal of Marital and Family Therapy, 26(1), 1-7.

Prochaska, J. O., Diclements, C., \& Norcross J. C. (1992). In search of how people change. American Psychologist, 47(9), 1102-1114.

Rosenzweig, S. A. (1954). A transvaluation of psychotherapy - a reply to Hans Eysenck. Journal of Abnormal and Social Psychology, 49, 298-304.

Shadish, W. R., Montgomery, L. M., Wilson, P., Wilson, M. R., Bright, I., \& Okuwumabua, T. (1993). Effects of family and marital psychotherapies: a meta-analysis. Journal of Consulting and Clínical Psychology, 61(6), 9921002.

Sloane, R. B., Staples, F. R., Cristol, A. H., Yorkston, N. J., \& Whipple, K. (1975). Short term analytical oriented psychotherapy vs. behavior therapy. Cambridge: Harvard University Press.

Smith, M. L., Glass, G. V., \& Miller, T. I. (1980). The benefits of psychotherapy. Baltimore: Johns Hopkins University Press.

Society of Clinical Psychology, American Psychological Association - Division 12 (2003). A guide to beneficial psychoterapy. Website http://www.apa.org/ divisions/div12/rev_est/, acessado em 23 de maio de 2003.

Stricker, G. (1992). The relationship of research to clínical practice. American Psychologist, 47(4), 543-549.

Wachtel, P. L. (1981). Psychoanalysis and behavior therapy: toward an integration. Nova York: Plenun.

Weil, P. (1978). Fronteiras da evolução e da morte. Petrópolis: Vozes.

${ }^{1}$ Texto original em inglês; tradução dos autores, como nos demais casos semelhantes a este.

${ }^{2}$ Texto original em espanhol - tradução dos autores.

Orestes Diniz Neto, doutor em Psicologia Clínica pela Pontifícia Universidade Católica do Rio de Janeiro, é professor no departamento de Psicologia da Universidade Federal de Minas Gerais. Endereço para correspondência: Av. Antônio Carlos, 6627 (Pampulha); Belo Horizonte, MG; CEP 31270-901. Tel.: (31) 34995027. Fax: (31) 3499-5027.E-mail: orestesd@ufmg.br

Terezinha Féres-Carneiro, doutora em Psicologia Clínica pela Pontifícia Universidade Católica de São Paulo, é Professora Titular no Departamento de Psicologia, Pontifícia Universidade Católica do Rio de Janeiro. Email: teferca@psi.puc-rio.br 